\title{
Comparison of hematological and inflammatory mortality predictors between older and younger COVID-19 patients
}

\author{
Suna AVCI ${ }^{1, \star_{\odot}}$, Vildan GURSOY ${ }^{\circledR}$
}

\begin{abstract}
${ }^{1}$ Department of Geriatrics, University of Health Sciences, Bursa Yuksek Ihtisas Education and Research Hospital, 16310 Bursa, Turkey

${ }^{2}$ Department of Hematology, University of Health Sciences, Bursa Yuksek Ihtisas Education and Research Hospital, 16310 Bursa, Turkey
\end{abstract}

\section{*Correspondence}

sunavci012@yahoo.com

(Suna AVCI)

\begin{abstract}
Background/objective: Several hematological and inflammatory parameters so far have been associated with COVID-19 disease severity; however, such evidence for particularly vulnerable elderly patients is lacking. This study aimed to investigate potential and practical biomarkers that could assist in predicting mortality at the presentation in a group of elderly and non-elderly patients.

Methods: This retrospective cohort study included 1820 COVID-19 patients hospitalized for treatment. Clinical and mortality data as well as certain hematological and inflammatory parameters were retrieved from records. For analysis, patients were divided into two groups as geriatric (age $\geq 65$ years) and non-geriatric subjects. The associated factors of the parameters on mortality were examined separately for elderly and younger patients.

Results: Following multivariate analysis, high neutrophil count and high troponin $\mathrm{T}$ levels emerged as significant independent predictors of mortality in both geriatric patients and younger patients. Low and high monocyte count was associated with increased mortality risk for geriatric and younger patients, respectively. In the geriatric population, high ferritin levels and high RBC count was associated with increased risk, but increased eosinophil count was associated with decreased risk. Low lymphocyte count emerged as a predictor of mortality among younger patients.

Conclusion: Several hematological and inflammatory parameters and indices may assist in predicting the mortality risk in patients with COVID-19; however, there appears to be some differences in terms of these predictors of mortality between elderly and younger patients. Larger prospective studies are warranted to support these findings.
\end{abstract}

\section{Keywords}

Elderly patients; COVID-19; SARS-CoV-2; Hematologic parameters; Predictors; Mortality

\section{Introduction}

The severe respiratory syndrome coronavirus-2 (SARS-CoV2) disease that originated in Wuhan China in December 2019 acquired the character of a global health problem [1]. Based on data provided by the World Health Organization, there were more than 178 million confirmed cases and almost 3.8 million deaths due to this disease as of 22 June 2021 [2], indicating a high mortality rate.

While the reported mortality rate was $2.3 \%$ in China, this figure has risen up to $7.2 \%$ in other countries such as Italy [3]. In addition, a higher mortality rate due to SARS-CoV2 infection has been reported among elderly populations [35]. In Turkey, the first case was documented on 11 March 2020 , and the reported mortality rates among those $>60$ years of age were $18 \%$ and $6 \%$ in inpatient or outpatient settings, respectively [6].
There clearly exists a need for biological markers that will allow clinicians to monitor the disease progression as well as to identify patients at an increased risk of complications or death [7]. A number of hematological parameters including white blood cell (WBC) count, lymphocyte count, neutrophil count, neutrophil/lymphocyte ratio (NLR), platelet count, eosinophil count as well as hemoglobin and several other biochemical parameters such as ferritin and troponin have been associated with COVID-19 disease severity [7, 8].

The need for reliable and easily accessible biological markers that will predict the clinical progression of COVID-19 is even more pronounced for geriatric patients. Thus, this study was undertaken to investigate potential and practical biomarkers that could assist in predicting the mortality at the presentation in a group of elderly and non-elderly patients. 


\section{Patients and method}

\subsection{Participants}

This retrospective cohort study was performed by the inclusion of COVID-19 patients admitted to and hospitalized at our reference pandemic hospital between 20 March 2020 and 15 May 2020. Demographic data, polymerase chain reaction (PCR) and imaging results, treatments administered, duration of hospital stay, mortality status, need for intensive care unit admission, and test results for hematological and inflammatory markers was retrieved from the hospital database. COVID19 diagnosis was based on the "Interim Guidelines" issued by the Turkish Ministry of Health. Thorax CT images of patients with negative PCR tests were compatible with COVID Pneumonia. Besides this, the clinical and laboratory of these patients were examined by the chest-infectious diseases team and these patients were named as COVID-19 in accordance with the general literature. Patients with positive PCR test or with suspected COVID-19 infection (PCR negative patients with clinical suspicion of COVID-19 and/or presence of COVID-19-specific lung involvement in thorax Computed tomography (CT)) who were treated at the hospital were included. Outpatients who could apply our center were accepted. Patients who needed ICU at the time of admission were excluded from the study. According to the diagnostic criteria published by the Ministry of Health, ICU admission criteria were as follows: dyspnea and respiratory distress; respiratory rate $>30 / \mathrm{min}, \mathrm{PaO}_{2} / \mathrm{FiO}_{2}<300 ; \mathrm{SPO}_{2}<90$ despite $5 \mathrm{~L} / \mathrm{min}$ oxygen treatment, $\mathrm{PaO}_{2}<70$, hypotension (systolic blood pressure $<90 \mathrm{mmHg}$ and/or more than $40 \mathrm{mmHg}$ drop and mean arterial pressure $<65 \mathrm{mmHg}$ ), tachycardia $>100 / \mathrm{min}$, acute kidney injury, development of acute organ dysfunction, patients with immunosuppression, acute bleeding diathesis, troponin increase, arrhythmia, lactate $>2 \mathrm{mmol}$, capillary return disorder, and cutis marmaratus were transferred to ICU. Patients were divided into two groups as geriatric (age $\geq 65$ years) and non-geriatric subjects for data analysis. Patients were excluded if they were under 18 years of age, received outpatient treatment, or had missing data. The study protocol was approved by local ethics committee of University of Health Science Bursa Yuksek Ihtisas Education and Research Hospital (date, 13 May 2020; no, 2011-KAEK-25 2020/05-19) and the study was conducted in accordance with the Declaration of Helsinki. In addition, the study protocol was approved by the Turkish Ministry of Health for COVID-19 research. This study used the data collected during routine clinical practice for COVID-19 patients and the data were handled anonymously.

\subsection{Patient management}

Only patients receiving inpatient care at normal wards or intensive care unit were included. At the time of study period, based on national therapeutic guidelines, Hydroxychloroquine \pm Azithromycin was given to patients with mild pneumonia, while Hydroxychloroquine and/or Favipiravir \pm Azithromycin was given to patients with severe pneumonia. For those with worsening clinical signs or pneumonia while receiving Hydroxychloroquine, Favipiravir was added to ongoing treatments. Oseltamivir was given to selected patients, since the study period coincided with the influenza season. Tocilizumab was administered to patients who develop Macrophage Activation Syndrome (MAS). Low molecular weight heparin (LMWH) was given to all patients unless contraindicated. Convalescent plasma therapy was administered to those with high fever lasting $>7$ days, $50 \%$ increase in lung infiltration after 24-48 hours, need for intensive care unit admission, need for vasopressors, and need for mechanical ventilation. Patients were admitted to intensive care unit if respiratory rate was $>30 / \mathrm{min}, \mathrm{PaO} 2 / \mathrm{FiO}_{2}$ was $<300$, or $\mathrm{SpO}_{2}$ was $<90 \%$ or $\mathrm{PaO}_{2}$ was $<70 \mathrm{mmHg}$ despite oxygen at a flow rate of 5 $\mathrm{L} / \mathrm{min}$. Mechanical ventilation with intubation was initiated in case of increased respiratory workload (dyspnea, tachypnea $\geq 30 / \mathrm{min}$ ), use of accessory respiratory muscles, paradoxical respiration, and respiratory alkalosis $(\mathrm{pH}>7.45)$.

\subsection{Assessments}

Complete blood count (CBC), serum biochemistry including C-reactive protein (CRP), D-dimer, troponin $\mathrm{T}$, ferritin, and fibrinogen, and erythrocyte sedimentation rate (ESR) were tested at admission. CBC-Mindray BC-6800, serum biochemistry-cobas c 702, CRP-Siemens BNII, troponin-cobas e 602, ESR-Vacuplus ESR100, Ddimer and fibrinogen-sysmex CS-5100 equipments were used for testing. In addition, previously reported derived parameters (lymphocyte/monocyte, neutrophil/lymphocyte, platelet/lymphocyte, dNLR, CRP/lymphocyte) or those with potential prognostic value based on our analysis (neutrophil/eosinophil, neutrophil/monocyte, troponin/eosinophil, ferritin/ eosinophil, neutrophil x troponin, neutrophil x ferritin) were estimated. Clinical variables such as duration of hospitalization, ICU admission, and mortality were recorded. The associated factors of hematological and inflammatory parameters on mortality were analyzed separately for elderly and younger patients.

\subsection{Statistical analysis}

IBM SPSS Statistics version 21.0 software (SPSS Inc., Chicago, IL) was used for the analysis of data. Descriptive data are presented in number (percentage) or mean \pm standard deviation, where appropriate. Categorical variables were compared using Pearson's chi-square test or Fisher's exact test. Depending on the normality of the data, continuous variables were compared using Mann-Whitney U or student's $t$ test for independent samples. Both hypothesis tests and graphical methods were used to test normality. Logistic regression was done for univariate and multivariate analysis of hematological/inflammatory parameters for their association with mortality. For the indices derived based on the emerging significant independent predictors and previously published studies, receiver operator characteristic (ROC) curves were generated and area under curve (AUC) values were calculated to examine the predictive role of these continuous parameters for mortality. Optimal cut-off values were identified and reported with their sensitivity and specificity for predicting mortality. Two-sided $p$ values $<0.05$ were considered indication of statistical significance. 
T A B L E 1. Demographical and clinical characteristics of the patients by age group.

\begin{tabular}{|c|c|c|c|c|}
\hline Patient characteristic & All patients & Age $\geq 65$ & Age $<65$ & $p$ \\
\hline & $\mathrm{n}=1820$ & $\mathrm{n}=578(31.8 \%)$ & $\mathrm{n}=1242(68.2 \%)$ & \\
\hline \multicolumn{5}{|l|}{ Demographics } \\
\hline Age (year), mean \pm SD & $53.6 \pm 19.1$ & $76.1 \pm 7.4$ & $43.2 \pm 12.9$ & $<0.001$ \\
\hline Female gender & $914(50.2 \%)$ & $301(52.1 \%)$ & $613(49.4 \%)$ & 0.280 \\
\hline PCR confirmed disease & $807(44.3 \%)$ & $161(27.9 \%)$ & $646(52.0 \%)$ & $<0.001$ \\
\hline \multicolumn{5}{|l|}{ Medications } \\
\hline Hydroxychloroquine & $1813(99.7 \%)$ & $575(99.7 \%)$ & $1238(99.8 \%)$ & 0.655 \\
\hline Azithromycin & $1191(65.7 \%)$ & $402(70.0 \%)$ & $789(63.7 \%)$ & 0.008 \\
\hline Enoxaparin & $1143(62.8 \%)$ & $346(60.0 \%)$ & $797(64.2 \%)$ & 0.084 \\
\hline Oseltamivir & $904(50.1 \%)$ & $266(46.9 \%)$ & $638(51.5 \%)$ & 0.068 \\
\hline Favipiravir & $222(12.3 \%)$ & $103(18.2 \%)$ & $119(9.6 \%)$ & $<0.001$ \\
\hline Plasma & $12(0.7 \%)$ & $6(1.0 \%)$ & $6(0.5 \%)$ & 0.213 \\
\hline Tocilizumab & $8(0.4 \%)$ & $2(0.4 \%)$ & $6(0.5 \%)$ & 1.000 \\
\hline \multicolumn{5}{|l|}{ Outcomes } \\
\hline Hospitalization (days), mean \pm SD & $6.7 \pm 5.1$ & $7.9 \pm 6.7$ & $6.1 \pm 3.9$ & $<0.001$ \\
\hline Mortality & $112(6.2 \%)$ & $85(14.7 \%)$ & $27(2.2 \%)$ & $<0.001$ \\
\hline ICU admission & $95(5.2 \%)$ & $73(12.8 \%)$ & $22(1.8 \%)$ & $<0.001$ \\
\hline Prolonged hospitalization* & $772(42.4 \%)$ & $294(50.9 \%)$ & $478(38.5 \%)$ & $<0.001$ \\
\hline
\end{tabular}

Unless otherwise stated, data presented as $n(\%)$. Since data is missing in a small number of patients, valid percentages are shown. *>5 days. Abbreviations: ICU, intensive care unit; SD, standard deviation.

\section{Results}

\subsection{Patient characteristics}

Table 1 shows demographical and clinical characteristics of the patients by age groups. Of 1820 patients, 807 (44.3\%) had PCR confirmed COVID-19 infection. Almost one third of the patients were in the geriatric age group. PCR confirmed infection was significantly less common among geriatric patients $(<0.001)$. The two groups did not differ in terms of gender distribution. Azithromycin and Favipiravir use were more common among geriatric patients. Mortality, ICU admission, and prolonged hospitalization rates were higher, and duration of hospitalization was longer in geriatric patients when compared to the patients younger than 65 years of age. Table 2 shows hematological/inflammatory parameters on admission by age group. Geriatric patients had significantly low hematocrit, hemoglobin, red blood cell, basophil, lymphocyte, and platelet counts ( $p<0.05$ for all). On the other hand, white blood cell, neutrophil, and monocyte counts, as well as ESR, CRP, troponin T, fibrinogen, and d-dimer levels were higher in the geriatric age group ( $p<0.05$ for all).

\subsection{Predictors of mortality}

Table 3 shows the predictors of mortality, which showed some variation across the two age groups. Following multivariate analysis, high neutrophil count and high troponin $\mathrm{T}$ levels emerged as significant independent predictors of mortality in both geriatric patients and younger patients. Low and high monocyte count was associated with increased mortality risk for geriatric and younger patients, respectively. In the geriatric population, high ferritin levels and high RBC count was associated with increased risk but per unit increase in eosinophil count was associated with decreased risk. On the other hand, low lymphocyte count emerged as one of the significant independent predictors of mortality among younger patients.

\subsection{Indices for mortality}

Indices derived based on the emerging significant independent predictors and previously published studies are shown on Table 4 with their respective AUC values to predict mortality. In addition, optimal cut-off values for those indices with an AUC $>0.800$ are also provided along with their respective sensitivities and specificities. Indices with the highest AUC values were troponin/eosinophil and ferritin/eosinophil for the geriatric age; and neutrophil $x$ ferritin and neutrophil/lymphocyte for younger patients. ROC curves are provided for these indices in Fig. 1.

\section{Discussion}

The findings of this study comparing a number of hematological variables in a large sample of elderly and non-elderly COVID-19 patients suggest that distinct sets of hematological parameters measured at presentation may have predictive value for mortality in these two different patient groups. To the best of our knowledge, the comparative prognostic value of hematological variables in geriatric vs. younger populations has not been subject to previous research. 
T A B L E 2. Hematological/inflammatory parameters on admission by age group.

\begin{tabular}{|c|c|c|c|}
\hline Parameter & Age $\geq 65$ & Age $<65$ & $p$ \\
\hline & $\mathrm{n}=578(31.8 \%)$ & $\mathrm{n}=1242(68.2 \%)$ & \\
\hline Hemoglobulin, g/dL & $11.7 \pm 2.1$ & $12.9 \pm 1.9$ & $<0.001$ \\
\hline Hematocrit, \% & $35.2 \pm 5.9$ & $38.8 \pm 5.3$ & $<0.001$ \\
\hline Red blood cell count $\left(\times 10^{6}\right.$ cells $\left./ \mu \mathrm{L}\right)$ & $4.1 \pm 0.8$ & $4.6 \pm 0.9$ & $<0.001$ \\
\hline White blood cell count $\left(\times 10^{3}\right.$ cells $\left./ \mu \mathrm{L}\right)$ & $9.0 \pm 6.7$ & $7.4 \pm 4.8$ & $<0.001$ \\
\hline Neutrophil count $\left(\times 10^{3}\right.$ cells $\left./ \mu \mathrm{L}\right)$ & $6.4 \pm 4.5$ & $4.6 \pm 3.4$ & $<0.001$ \\
\hline Eosinophil count $(\times$ cells $/ \mu \mathrm{L})$ & $136.8 \pm 153.6$ & $144.4 \pm 169.5$ & 0.243 \\
\hline Basophil count $(\times$ cells $/ \mu \mathrm{L})$ & $30.7 \pm 21.5$ & $36.2 \pm 109.2$ & $<0.001$ \\
\hline Lymphocyte count $\left(\times 10^{3}\right.$ cells $\left./ \mu \mathrm{L}\right)$ & $1.7 \pm 1.4$ & $2.0 \pm 0.9$ & $<0.001$ \\
\hline Monocyte count $\left(\times 10^{3}\right.$ cells $\left./ \mu \mathrm{L}\right)$ & $0.6 \pm 0.3$ & $0.5 \pm 0.3$ & $<0.001$ \\
\hline Platelet count $\left(\times 10^{3}\right.$ cells $\left./ \mu \mathrm{L}\right)$ & $233.5 \pm 89.7$ & $246.7 \pm 87.5$ & $<0.001$ \\
\hline C-reactive protein, $\mathrm{mg} / \mathrm{L}$ & $55.3 \pm 61.4$ & $31.1 \pm 49.1$ & $<0.001$ \\
\hline $\mathrm{ESR}, \mathrm{mm} / \mathrm{h}$ & $55.5 \pm 28.9$ & $40.1 \pm 26.3$ & $<0.001$ \\
\hline Troponin $\mathrm{T}, \mathrm{pg} / \mathrm{mL}$ & $75.3 \pm 244.9$ & $25.2 \pm 348.6$ & $<0.001$ \\
\hline Ferritin, ng/mL & $325.1 \pm 993.7$ & $208.6 \pm 307.1$ & 0.05 \\
\hline Fibrinogen, $\mathrm{mg} / \mathrm{dL}$ & $514.1 \pm 172.8$ & $437.9 \pm 162.8$ & $<0.001$ \\
\hline D-dimer, $\mu \mathrm{g} / \mathrm{mL}$ & $3.2 \pm 13.2$ & $1.2 \pm 3.5$ & $<0.001$ \\
\hline
\end{tabular}

Data presented as mean \pm standard deviation. ESR, erythrocyte sedimentation rate.

TA B L E 3. Multivariate analysis of the hematological/inflammatory parameters on admission for mortality.

\begin{tabular}{lcc} 
& Age $\geq 65$ & Age $<65$ \\
High RBC count $\left(>5.03 \times 10^{6}\right.$ cells $\left./ \mu \mathrm{L}\right)$ & $2.70(1.02-7.14), p=0.045$ & \\
High neutrophil count $\left(>8 \times 10^{3}\right.$ cells $\left./ \mu \mathrm{L}\right)$ & $5.56(2.95-10.48), p<0.001$ & $18.74(6.62-53.06), p<0.001$ \\
\hline Eosinophil count $(\operatorname{cells} / \mu \mathrm{L}) \dagger$ & $0.994(0.990-0.998), p=0.001$ & \\
\hline Low monocyte count $\left(<0.3 \times 10^{3}\right.$ cells $\left./ \mu \mathrm{L}\right)$ & $3.76(1.78-7.93), p=0.001$ & \\
High monocyte count $\left(>0.9 \times 10^{3}\right.$ cells $\left./ \mu \mathrm{L}\right)$ & & $3.25(1.08-9.75), p=0.036$ \\
Low lymphocyte count $\left(<0.9 \times 10^{3}\right.$ cells $\left./ \mu \mathrm{L}\right)$ & & $10.98(4.12-29.29), p<0.001$ \\
\hline High troponin $\mathrm{T}(>14 \mathrm{pg} / \mathrm{mL})$ & $16.22(1.98-132.69), p=0.009$ & $17.90(4.23-72.36), p<0.001$ \\
High ferritin $(>150 \mathrm{ng} / \mathrm{mL})$ & $3.74(1.02-13.70), p=0.046$ & \\
\hline
\end{tabular}

Data presented as odds ratio (95\% confidence interval), $p$ value. $\dagger$ Odds ratios are for per unit increase. $R B C$, red blood cell.

On the other hand, the association between hematologic parameters and prognosis, need for intensive care admission, and mortality in COVID-19 was examined in many previous studies in the general population. In four recent metaanalyses including geriatric patients, low lymphocyte count, low platelet count, and high neutrophil count were found to correlate significantly with poor prognosis, severe disease course, ICU admission, and mortality [9-12]. In one of these meta-analyses, a cut-of value of $3.74 \times 10^{3} / \mathrm{L}$ for the neutrophil count was reported to indicate increased risk of severe COVID-19 disease [11]. In another study, thrombocytopenia at presentation was found to be associated with a 3 -fold increase in mortality, as compared to those without thrombocytopenia [13]. In our study, we observed that low RBC, hemoglobin, hematocrit, basophil count, lymphocyte count, and platelet count as well as high neutrophil, WBC, and monocyte count were associated with increased mortality from COVID-19 infection in the elderly. Therefore, our geriatric patients represent a high-risk group also when hematologic parameters were considered, since they had such impairments at the time of admission. In our study, we determined that there were low lymphocytes and platelets, and high neutrophil, wbc, and monocytes in the geriatric group. Therefore, our elderly patient group is at risk for poor outcome in terms of hematological parameters.

It is not surprising to observe increased inflammation in patients with poor clinical status, when one considers the role of the cytokine storm in severe COVID-19 infection. In previous reviews and studies investigating different biomarkers that could be potentially associated with a more severe disease course in COVID-19 infection, elevation of several inflammatory parameters such as CRP, ferritin, and ESR were 
TA B L E 4. Potential indices for the prediction of mortality.

\begin{tabular}{|c|c|c|c|c|}
\hline & \multicolumn{2}{|c|}{ Age $\geq 65$} & \multicolumn{2}{|c|}{ Age $<65$} \\
\hline & AUC $(95 \% \mathrm{CI})$ & Cut-off $(\mathrm{Se} / \mathrm{Sp})$ & AUC $(95 \% \mathrm{CI})$ & Cut-off (Se/Sp) \\
\hline Neutrophil/eosinophil & $0.822(0.777-0.867)$ & $0.29(70 / 81)$ & $0.702(0.579-0.824)$ & - \\
\hline Neutrophil/monocyte & $0.815(0.761-0.869)$ & $12.7(72 / 78)$ & $0.771(0.660-0.882)$ & - \\
\hline Troponin/eosinophil & $0.857(0.820-0.893)$ & $0.40(97 / 64)$ & $0.829(0.752-0.907)$ & $0.34(83 / 83)$ \\
\hline Ferritin/eosinophil & $0.865(0.817-0.914)$ & $5.25(80 / 81)$ & $0.833(0.734-0.931)$ & $1.45(94 / 58)$ \\
\hline Neutrophil $\mathrm{x}$ troponin & $0.817(0.766-0.868)$ & $206(80 / 75)$ & $0.828(0.700-0.957)$ & $16.5(88 / 63)$ \\
\hline Neutrophil x ferritin & $0.828(0.768-0.888)$ & $1220(73 / 78)$ & $0.905(0.820-0.990)$ & $1637(89 / 89)$ \\
\hline Lymphocyte/monocyte & $0.343(0.272-0.415)$ & - & $0.221(0.092-0.350)$ & - \\
\hline Neutrophil/lymphocyte & $0.813(0.760-0.867)$ & $4.85(78 / 73)$ & $0.846(0.730-0.962)$ & $2.6(89 / 71)$ \\
\hline Platelet/lymphocyte & $0.645(0.574-0.717)$ & - & $0.642(0.501-0.784)$ & - \\
\hline dNLR & $0.818(0.764-0.872)$ & $3.0(77 / 73)$ & $0.765(0.624-0.906)$ & - \\
\hline CRP/lymphocyte & $0.794(0.741-0.848)$ & - & $0.808(0.698-0.919)$ & $8.6(83 / 61)$ \\
\hline
\end{tabular}

AUC data presented as AUC (95\% confidence interval), $p$ value. Cut-off data provided for parameters with an AUC higher than 0.800. AUC, area under curve; Se/Sp, sensitivity/specificity in percent; RBC, red blood cell; $C R P$, c-reactive protein; $d N L R$, derived neutrophil lymphocyte ration defined as neutrophil count divided by the result of white blood cell count minus neutrophil count.

found to be linked with increased likelihood of more severe disease [14-18]. Based on their univariate analysis, Zhou, et al. [19] reported an association between high serum ferritin and death, but no multivariate analysis results were presented by the authors.

In addition, some studies suggested that low lymphocyte count as well as low eosinophil as positive indicators for diagnosis in COVID-19 [20]. For example, Liu et al. [21], in their COVID-19 patients, observed low eosinophil counts at admission that returned to normal before discharge, concluding that increasing eosinophil levels may represent an indicator of clinical improvement. On the other hand, a systematic literature review suggested that eosinopenia is not a reliable marker for COVID-19 prognosis [22]. Although low eosinophil count emerged as a predictor of mortality only in our elderly patients, several indices derived from eosinophil counts such as troponin/eosinophil and ferritin/eosinophil ratio were found to have a predictive value for both age groups.

Several previous publications found an association of Ddimer with severe COVID-19 disease and mortality [23]. Ddimer, which is elevated in the presence of thrombotic events, has also been reported to be a significant prognostic factor in COVID-19 disease, and in a review of 19 articles, a cut-off level of $0.4 \mu \mathrm{g} / \mathrm{mL}$ was proposed for D-dimer levels [15]. In another systematic review, a 3- to 4-fold increase in D-dimer levels were found to be related with poor prognosis in these patients [24].

Elevated troponin levels have been associated with multiorgan failure [12], and in autopsy series COVID-19 has been shown to cause systemic cardiovascular damage via myocyte injury and hyperinflammation [25]. Therefore, it is not surprising to observe increased troponin levels in COVID-19 patients [26]. Again, increased troponin was found to be associated with higher risk of mortality in both age groups in the current study.
Neutrophil/lymphocyte ratio (NLR) was reported to be an independent risk factor for the severity of COVID-19 infection [27-29]. In one study involving 1004 patients, patients with severe infection had 5-fold higher NLR, and 40 of the deaths occurred in those over 62 years of age [30]. In a large metaanalysis, NLR was found to be significantly increased levels in the severe and non-survivor group [31]. In a study evaluating the severe and fatal forms of the disease, NLR was found to have a high prognostic value (AUROC: 0.831) and the predict of mortality cut-off value was determined as 7.4 (se $=75 \%, \mathrm{sp}=84 \%$ ) [32]. In our study conducted with larger series, the mortality cut-off value was seen 4.85 ( $\mathrm{se}=78 \%$, $\mathrm{sp}=73 \%)$ and $2.6(\mathrm{se}=78 \%, \mathrm{sp}=73 \%)$ respectively, in the geriatric and young groups, separated by age groups. In another study, the predict of mortality NLR cut-off value $4.7(\mathrm{se}=72 \%, \mathrm{sp}=51 \%$ ) was found similar to our study [33]. Also in another different study, in which people over the age of 50 were given, the cut off value was found to be 3.3. [34]. Also, there have been reports regarding high monocyte/lymphocyte ratio (MLR), where this parameter was found to indicate cardiovascular risk in patients with severe pneumonia. In that study, patients with higher MLR had imaging signs with poor progression [35]. Again in another study, increased platelet/lymphocyte ratio (PLR) was linked with increased duration of hospital stay. A pronounced elevation in platelets also correlated with longer average length of hospital study and this was explained based on cytokine storm [36]. One study reported a tendency for lower lymphocyte, higher leukocyte, higher NLR, and lower monocyte, eosinophil, and basophil percentages among severe cases [37]. While a number of different hematologic indices derived from neutrophils, eosinophils, monocytes, and lymphocytes were found to be predictors of mortality in elderly and/or younger COVID-19 patients, we failed to observe a similar finding for platelet count and indices derived from this parameter. This 

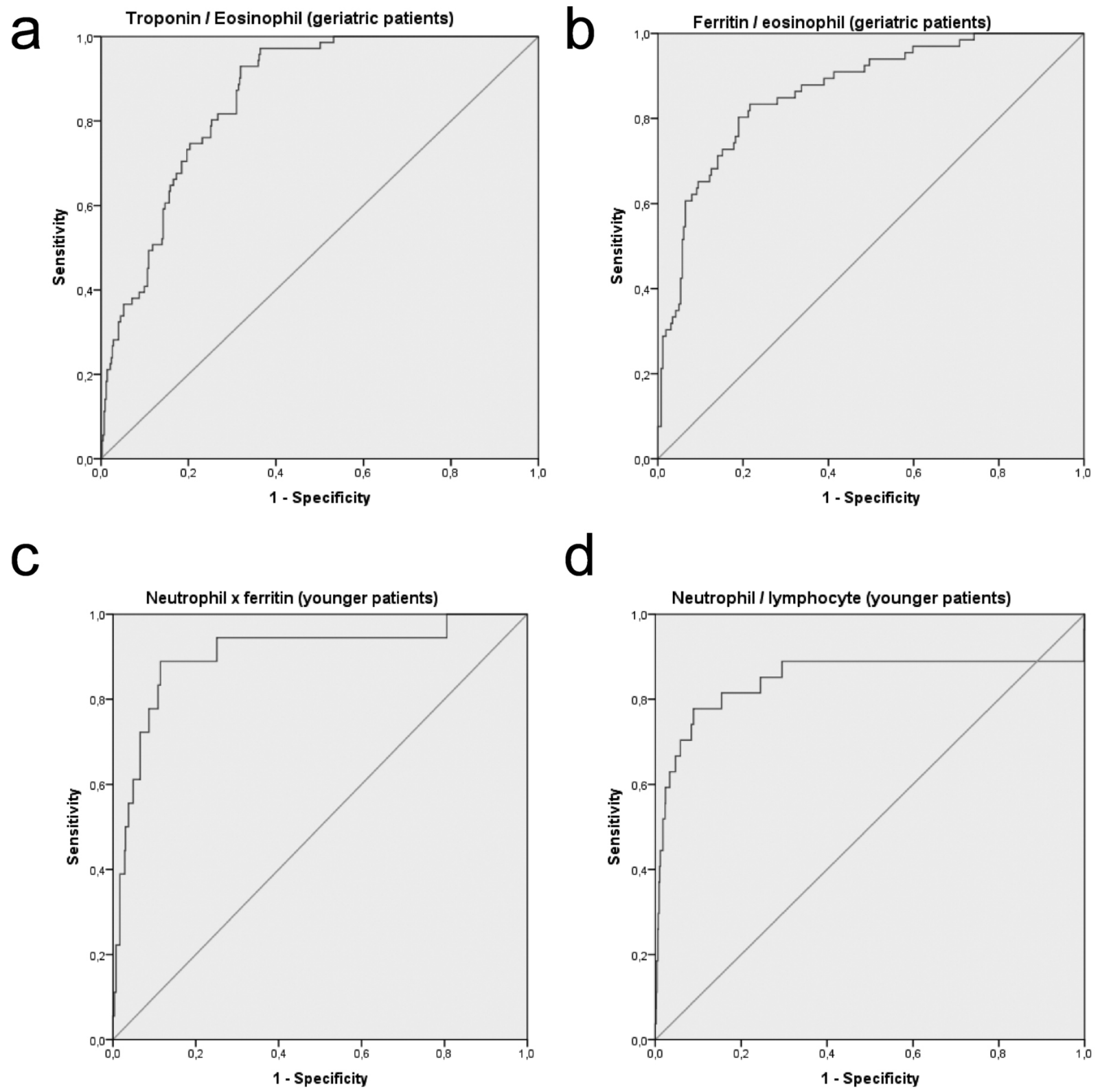

F I G URE 1. Receiver operator characteristics curves for selected indices for the prediction of mortality. (a) troponin eosinophil ratio in the geriatric age group, (b) ferritin eosinophil ratio in the geriatric age group, (c) neutrophil multiplied by ferritin in younger patients, (d) neutrophil lymphocyte ratio in younger patients.

observation suggests that rather than the platelet count at presentation, platelet counts in subsequent course of the disease may be more important in terms of predicting mortality, and this issue requires further studies. In line with the published data, NLR was a predictor of mortality in both age groups in the present study. However, since the young group had the highest AUC, it was seen that it should be emphasized in patient evaluation compared to the older group. Besides this, neutrophil $x$ ferritin indices in the young group not discussed in the literature, and troponin/eosinophil and ferritin/eosinophil indices in the geriatric group are encountered in our study when compared to age groups.

Our study distinct certain differences between elderly and younger patients in terms of predictors of mortality. In the light of the treatment guide prepared by the Ministry of Health at that time, Favipiravir was recommended for severe COVID-19 patients and Azithromycin for secondary infections that may develop in the course of COVID-19. This is an indication that COVID-19 causes more complications in the geriatric patients.

It is believed that COVID-19 may impair hematopoiesis by directly infecting the bone marrow precursors or may induce an auto-immune response against blood cells and a cytokine storm. This may lead to systemic inflammation, and even to multiple organ injury [38, 39]. Furthermore, age-related changes are known to occur in hematopoietic tissues and immunosenescence is known to develop over time in an individual's lifetime. In addition to cellular senescence, nutritional deficiencies due to reduced intake and absorption or losses may cause cellular and functional impairment in leukocyte components, increasing the vulnerability toward disease in the elderly population [40]. Therefore, age-related cellular changes generally increase the predisposition to infections 
among geriatric populations. However, although COVID19 has a more deadly course in the elderly, the differential responses between older and younger bone marrows in this infection have not been thoroughly understood.

Defined as SARS-CoV-2 receptor, Angiotensin-converting enzyme 2 (ACE2) receptor levels in cardiac and pulmonary tissues differ in young and geriatric groups [41]. In addition, the cause of developing lymphopenia is thought to be lympholysis caused by viral invasion of lymphocytes due to the ACE-2 gene on lymphocytes [42]. Neutrophil increase is the first expected cellular response in infection. In this case, the increase in NLR in COVID-19 can be expected to be related to poor prognosis. The association of low monocyte or eosinophils counts with increased mortality in the elderly may actually result from related mechanisms. In our study, contrary to the observation that monocytosis at presentation was associated with increased mortality among the younger individuals, low monocyte count was related with increased mortality in the elderly. This may be due to the presence of a more robust monocyte/macrophage activation cascade among younger patients in relation to inflammation. In one previous study comparing bone marrow responses in younger and elderly individuals upon exposure to COVID19, a reprogrammed immune landscape was emphasized in the elderly. It has also been proposed that elderly individuals may have an upregulation of inflammatory genes via altered immunomodulation mechanisms [43], which may potentially lead to an increased inclination for cytokine storm in this population. Also, the predictive value of ferritin in mortality is suggestive of a harbinger role of ferritin for cytokine storm. Also, increased RBC counts in the elderly may be related with the predisposition to hemoconcentration among these subjects compared to younger individuals.

One major limitation of our study is its retrospective nature. And also, in our study, only outpatients were included, and serious patients who needed ICU at the time of admission were excluded. However, the comorbidities of the patients were not included in the study. Future prospective studies conducted in a more organized setting would provide valuable information. The pathological mechanisms underlying the alterations in hematologic parameters observed in the course of COVID-19 infection are not clearly understood, indicating the need for further clinical and basic investigations.

\section{Conclusions}

Better characterization of hematological, biochemical, and inflammatory parameters that can predict mortality in geriatric patients, who have emerged as one of the most vulnerable groups since the start of the pandemic, will prove to be clinically useful.

Although there were some differences in terms of the predictors of mortality between elderly and younger patients, this study demonstrated that a number of hematological and inflammatory parameters and indices derived from these parameters on admission may assist in predicting the mortality risk in patients with COVID-19. The predictive value of these potential markers and indices may be further refined by monitoring their patterns over time in larger patient samples and in prospective setting.

\section{AUTHOR CONTRIBUTIONS}

SA, Literature search, data analysis, data interpretation, writing. VG, Literature search, data interpretation.

\section{ETHICS APPROVAL AND CONSENT TO PARTICIPATE}

The study protocol was approved by local ethics committee of University of Health Science Bursa Yuksek Ihtisas Education and Research Hospital (date, May 13, 2020; no, 2011-KAEK25 2020/05-19) and the study was conducted in accordance with the Declaration of Helsinki.

\section{ACKNOWLEDGMENT}

We would like to thank to Dr. Ali Erol and Dr. Melike Yazıcifor their valuable contribution, and nameless health care heros who sacrificed during Covid pandemic and Gökhan Kilıvan who always the best friend and statistics magician.

\section{FUNDING}

This research received no external funding.

\section{CONFLICT OF INTEREST}

The authors declare no conflict of interest.

\section{REFERENCES}

[1] Zhu N, Zhang D, Wang W, Li X, Yang B, Song J, et al. A novel coronavirus from patients with pneumonia in China, 2019. New England Journal of Medicine. 2020; 382: 727-733.

[2] World Health Organization. WHO Coronavirus Disease (COVID19) Dashboard. 2021. Available at: https://covid19.who.int/ (Accessed: 22 June 2021).

[3] Dhama K, Patel SK, Kumar R, Rana J, Yatoo MI, Kumar A, et al. Geriatric population during the COVID-19 pandemic: problems, considerations, exigencies, and beyond. Frontiers in Public Health. 2020; 8: 574198.

[4] Onder G, Rezza G, Brusaferro S. Case-fatality rate and characteristics of patients dying in relation to COVID-19 in Italy. Journal of the American Medical Association. 2020; 323: 1775-1776.

[5] Richardson S, Hirsch JS, Narasimhan M, Crawford JM, McGinn T, Davidson KW, et al. Presenting characteristics, comorbidities, and outcomes among 5700 patients hospitalized With COVID-19 in the New York City area. Journal of the American Medical Association. 2020; 323: 2052-2059.

[6] Esme M, Koca M, Dikmeer A, Balci C, Ata N, Dogu BB, et al. Older adults with coronavirus disease 2019; a nationwide study in Turkey. Journals of Gerontology, Series A: Biological Sciences and Medical Sciences. 2021; 76: e68-e75.

[7] Ponti G, Maccaferri M, Ruini C, Tomasi A, Ozben T. Biomarkers associated with COVID-19 disease progression. Critical Reviews in Clinical Laboratory Sciences. 2020; 57: 389-399.

[8] Zhang G, Zhang J, Wang B, Zhu X, Wang Q, Qiu S. Analysis of clinical characteristics and laboratory findings of 95 cases of 2019 novel coronavirus pneumonia in Wuhan, China: a retrospective analysis. Respiratory Research. 2020; 21: 74.

[9] Bao J, Li C, Zhang K, Kang H, Chen W, Gu B. Comparative analysis 
of laboratory indexes of severe and non-severe patients infected with COVID-19. Clinica Chimica Acta. 2020; 509: 180-194.

[10] Danwang C, Endomba FT, Nkeck JR, Wouna DLA, Robert A, Noubiap JJ. A meta-analysis of potential biomarkers associated with severity of coronavirus disease 2019 (COVID-19). Biomarker Research. 2020; 8: 37.

[11] Elshazli RM, Toraih EA, Elgaml A, El-Mowafy M, El-Mesery M, Amin MN, et al. Diagnostic and prognostic value of hematological and immunological markers in COVID-19 infection: a meta-analysis of 6320 patients. PLoS ONE. 2020; 15: e0238160.

[12] Henry BM, de Oliveira MHS, Benoit S, Plebani M, Lippi G. Hematologic, biochemical and immune biomarker abnormalities associated with severe illness and mortality in coronavirus disease 2019 (COVID-19): a metaanalysis. Clinical Chemistry and Laboratory Medicine. 2020; 58: 10211028.

[13] Liu Y, Sun W, Guo Y, Chen L, Zhang L, Zhao S, et al. Association between platelet parameters and mortality in coronavirus disease 2019: retrospective cohort study. Platelets. 2020; 31: 490-496.

[14] Tjendra Y, Al Mana AF, Espejo AP, Akgun Y, Millan NC, GomezFernandez C, et al. Predicting disease severity and outcome in COVID19 patients: a review of multiple biomarkers. Archives of Pathology \& Laboratory Medicine. 2020; 144: 1465-1474.

[15] Pourbagheri-Sigaroodi A, Bashash D, Fateh F, Abolghasemi H. Laboratory findings in COVID-19 diagnosis and prognosis. Clinica Chimica Acta. 2020; 510: 475-482.

[16] Liu W, Tao Z, Wang L, Yuan M, Liu K, Zhou L, et al. Analysis of factors associated with disease outcomes in hospitalized patients with 2019 novel coronavirus disease. Chinese Medical Journal. 2020; 133: 1032-1038.

[17] Ruan Q, Yang K, Wang W, Jiang L, Song J. Clinical predictors of mortality due to COVID-19 based on an analysis of data of 150 patients from Wuhan, China. Intensive Care Medicine. 2020; 46: 846-848.

[18] Tan C, Huang Y, Shi F, Tan K, Ma Q, Chen Y, et al. C-reactive protein correlates with computed tomographic findings and predicts severe COVID-19 early. Journal of Medical Virology. 2020; 92: 856862.

[19] Zhou F, Yu T, Du R, Fan G, Liu Y, Liu Z, et al. Clinical course and risk factors for mortality of adult inpatients with COVID-19 in Wuhan, China: a retrospective cohort study. The Lancet. 2020; 395: 1054-1062.

[20] Zhang J, Dong X, Cao Y, Yuan Y, Yang Y, Yan Y, et al. Clinical characteristics of 140 patients infected with SARS-CoV-2 in Wuhan, China. Allergy. 2020; 75: 1730-1741.

[21] Liu F, Xu A, Zhang Y, Xuan W, Yan T, Pan K, et al. Patients of COVID-19 may benefit from sustained Lopinavir-combined regimen and the increase of Eosinophil may predict the outcome of COVID-19 progression. International Journal of Infectious Diseases. 2020; 95: 183191.

[22] Lippi G, Henry BM. Eosinophil count in severe coronavirus disease 2019. QJM: an International Journal of Medicine. 2020; 113: 511-512.

[23] Tang N, Li D, Wang X, Sun Z. Abnormal coagulation parameters are associated with poor prognosis in patients with novel coronavirus pneumonia. Journal of Thrombosis and Haemostasis. 2020; 18: 844-847.

[24] Rostami M, Mansouritorghabeh H. D-dimer level in COVID-19 infection: a systematic review. Expert Review of Hematology. 2020; 13: 12651275.

[25] Hanley B, Lucas SB, Youd E, Swift B, Osborn M. Autopsy in suspected COVID-19 cases. Journal of Clinical Pathology. 2020; 73: 239-242.

[26] Duerr GD, Heine A, Hamiko M, Zimmer S, Luetkens JA, Nattermann J, et al. Parameters predicting COVID-19-induced myocardial injury and mortality. Life Sciences. 2020; 260: 118400.

[27] Xia X, Wen M, Zhan S, He J, Chen W. An increased neutrophil/lymphocyte ratio is an early warning signal of severe COVID-19. Nan Fang Yi Ke Da Xue Xue Bao 2020; 40: 333-336. (In Chinese)
[28] Liu Y, Du X, Chen J, Jin Y, Peng L, Wang HHX, et al. Neutrophilto-lymphocyte ratio as an independent risk factor for mortality in hospitalized patients with COVID-19. Journal of Infection. 2020; 81: e6e12.

[29] Yang A, Liu J, Tao W, Li H. The diagnostic and predictive role of NLR, d-NLR and PLR in COVID-19 patients. International Immunopharmacology. 2020; 84: 106504.

[30] Yan X, Li F, Wang X, Yan J, Zhu F, Tang S, et al. Neutrophil to lymphocyte ratio as prognostic and predictive factor in patients with coronavirus disease 2019: a retrospective cross-sectional study. Journal of Medical Virology. 2020; 92: 2573-2581.

[31] Mahat RK, Panda S, Rathore V, Swain S, Yadav L, Sah SP. The dynamics of inflammatory markers in coronavirus disease-2019 (COVID-19) patients: a systematic review and meta-analysis. Clinical Epidemiology and Global Health. 2021; 11: 100727.

[32] Sayah W, Berkane I, Guermache I, Sabri M, Lakhal FZ, Yasmine Rahali $\mathrm{S}$, et al. Interleukin-6, procalcitonin and neutrophil-to-lymphocyte ratio: Potential immune-inflammatory parameters to identify severe and fatal forms of COVID-19. Cytokine. 2021; 141: 155428.

[33] BG S, Gosavi S, Ananda Rao A, Shastry S, Raj SC, Sharma A, et al. Neutrophil-to-Lymphocyte, Lymphocyte-to-Monocyte, and Plateletto-Lymphocyte Ratios: Prognostic Significance in COVID-19. Cureus. 2021; 13: e12622.

[34] Liu J, Liu Y, Xiang P, Pu L, Xiong H, Li C, et al. Neutrophil-tolymphocyte ratio predicts critical illness patients with 2019 coronavirus disease in the early stage. Journal of Translational Medicine. 2020; 18: 206.

[35] Yang Z, Shi J, He Z, Lü Y, Xu Q, Ye C, et al. Predictors for imaging progression on chest CT from coronavirus disease 2019 (COVID-19) patients. Aging. 2020; 12: 6037-6048.

[36] Qu R, Ling Y, Zhang Y, Wei L, Chen X, Li X, et al. Platelet-to-lymphocyte ratio is associated with prognosis in patients with coronavirus disease- 19 . Journal of Medical Virology. 2020; 92: 1533-1541.

[37] Qin C, Zhou L, Hu Z, Zhang S, Yang S, Tao Y, et al. Dysregulation of Immune Response in Patients with Coronavirus 2019 (COVID-19) in Wuhan, China. Clinical Infectious Diseases. 2020; 71: 762-768.

[38] Cummings MJ, Baldwin MR, Abrams D, Jacobson SD, Meyer BJ, Balough EM, et al. Epidemiology, clinical course, and outcomes of critically ill adults with COVID-19 in New York City: a prospective cohort study. The Lancet. 2020; 395: 1763-1770.

[39] Henderson LA, Canna SW, Schulert GS, Volpi S, Lee PY, Kernan KF, et al. On the alert for cytokine storm: immunopathology in COVID-19. Arthritis \& Rheumatology. 2020; 72: 1059-1063.

[40] Al-Zahrani J. SARS-CoV-2 associated COVID-19 in geriatric population: a brief narrative review. Saudi Journal of Biological Sciences. 2021; 28: 738-743.

[41] Koff WC, Williams MA. Covid-19 and immunity in aging populations - a new research agenda. New England Journal of Medicine. 2020; 383: 804-805.

[42] Tan L, Wang Q, Zhang D, Ding J, Huang Q, Tang Y, et al. Lymphopenia predicts disease severity of COVID-19: a descriptive and predictive study. Signal Transduction and Targeted Therapy. 2020; 5: 33.

[43] Zheng Y, Liu X, Le W, Xie L, Li H, Wen W, et al. A human circulating immune cell landscape in aging and COVID-19. Protein \& Cell. 2020; 11: $740-770$.

How to cite this article: Suna AVCI, Vildan GURSOY. Comparison of hematological and inflammatory mortality predictors between older and younger COVID-19 patients. Signa Vitae. 2022;18(1):68-75. doi:10.22514/sv.2021.125. 